\title{
La construcción del lenguaje en Paraguay: fonologías, ortografías e ideologías en un país multilingüe
}

\author{
Jan David Hauck* \\ University of California, Los Angeles, EE. UU.
}

\begin{abstract}
Resumen
En este articulo se analiza una controversia sobre la inclusión de la lengua guaraní en el sistema de educación bilingüe en Paraguay. La controversia es el resultado de la compleja realidad socio-lingüística del país: El guaraní es lengua oficial y un emblema importante de la identidad nacional, pero también una lengua de estatus inferior al castellano, lo que se conoce como situación de "diglosia". Además, los hablantes tienen actitudes negativas sobre sus propias prácticas del habla, siendo caracterizadas por fenómenos de convergencia y mezcla. Esto, algunos estudiosos lo identificaron como "diglosia de segundo grado" entre prácticas discursivas cotidianas y la construcción ideológica del guaraní "real" de la comunidad del habla. En una medida controversial, planificadores lingüísticos del gobierno acreditaron una versión "híbrida" del guaraní que permitiría préstamos del español para remediar esta situación y facilitar la enseñanza del guaraní, acercando la versión oficial al habla cotidiano. Esta medida causó un debate incesante sobre la versión del guaraní
\end{abstract}

* Para correspondencia, dirigirse a: Jan David Hauck (jan.d.hauck@ucla.edu), Department of Anthropology, University of California, Los Angeles, 341 Haines Hall, Box 951553, Los Angeles, CA 90095, USA. 
que se debería usar en la enseñanza. Se utilizará este debate aquí para explorar las ideologías lingüísticas dominantes en el país, responsables tanto de las dificultades en la educación bilingüe como de las ambigüedades en el uso y en las actitudes acerca del guaraní.

Palabras clave: purificación, hibridación, lengua estándar, educación bilingüe, ideología lingüística, guaraní paraguayo.

\section{The construction of language in Paraguay: Phonologies, ORTHOGRAPHIES AND IDEOLOGIES IN A MULTILINGUAL COUNTRY}

Abstract

This paper analyzes a controversy about the inclusion of the Guaraní language in the bilingual education system of Paraguay. The controversy is the result of the country's complex sociolinguistic reality: Guaraní is an official language and an important emblem of national identity, but also a language of inferior status to Spanish, which is known as a situation of "diglossia". Moreover the speakers have negative attitudes towards their own speech practices, characterized by phenomena of convergence and mixing. This, some scholars identified as a "second order diglossia" between everyday discourse and the speech community's ideological construction of "real" Guaraní. In a controversial measure, government language planners have accredited a "hybrid" Guaraní version that would allow language borrowings from Spanish to remedy this situation and to facilitate the teaching of Guaraní, bringing the official version closer to everyday speech. This measure caused a heated debate about the version of Guaraní that should be used in education. The debate will be used here to explore the dominant language ideologies in the country that are responsible for the difficulties in bilingual education and for the ambiguities in the use of and attitudes towards Guaraní.

Keywords: purification, hybridization, standard language, bilingual education, language ideology, Paraguayan Guaraní.

Recibido: 04/09/14 Aceptado: 16/10/14 


\section{INTRODUCCIÓN ${ }^{1}$}

El Paraguay, como otros países que fueron "conquistados" y colonizados, ha sido objeto de fuertes arrebatos culturales. Que nuestro pueblo mantenga su lengua, es un fenómeno que demuestra nuestra lucha constante y sostenida de resistencia cultural.

Félix de Guarania (2008: 19)

El mantenimiento de la lengua guaraní en Paraguay es sinónimo de la resistencia a la asimilación cultural y la dominación extranjera. En los discursos sobre la identidad nacional se destaca frecuentemente el papel del guaraní en la lucha por la independencia y en las guerras de la Triple Alianza (1864-70) y del Chaco (1932-35): no solo como medio de comunicación y lengua secreta, sino que también como el símbolo unificador más importante, emblema de la cultura nacional paraguaya (Corvalán 1981; Steckbauer 1999). Según su mito de origen, la nación paraguaya tiene sus raíces en las poblaciones indígenas de la historia precolonial y hablar su lengua constituye una señal de continuidad cultural (Potthast 1999; Gynan 1998). En las palabras del poeta y escritor paraguayo Félix de Guarania (1924-2011): "Nadie puede dejar de reconocer que somos mestizos, que corre en nuestras venas sangre guaraní, nuestra alma pertenece a la guaraniedad - eso no se puede negar-, por eso hay que salvar el guaraní” (de Guarania 2008: 19).

Ciertamente, la historia y la situación actual del guaraní son bastante particulares: Paraguay es el único país latinoamericano donde se logró mantener una lengua indígena a pesar de 500 años de contacto con el castellano, lengua del colonizador, y esta lengua, el guaraní, no solo es hablada por la mayoría de la población, sino también reconocida como lengua oficial en todo su territorio, en condiciones iguales al castellano. Pero no es solo eso lo que hace a Paraguay un lugar privilegiado para estudiar las representaciones sociales del lenguaje. No obstante lo arriba señalado, Paraguay también se conoce en la literatura por su diglosia (Ferguson 1959), la asimetría en el estatus y uso de sus dos lenguas, reflejando la desigualdad socio-económica y de género discursivo (Fishman 1967; Melià 1973). De acuerdo con esta situación, el uso del guaraní está limitado a las áreas

Agradezco a Cristián Lagos sus valiosos comentarios y correcciones siendo yo el único responsable del uso que he hecho de las sugerencias y de cualquier error o malinterpretación. 
rurales y el sector más desfavorecido económicamente o a la comunicación informal; asimismo el castellano está asociado a la comunicación pública y formal y a los sectores de estatus socio-económico más alto en los centros urbanos (Gynan 1997, 2001). Por eso, muchos autores están de acuerdo con de Guarania (2008) en cuanto a que la lucha para el "rescate" del guaraní aún continúa. Sin embargo, las opiniones difieren sobre cómo lograrlo. Para algunos, lo que está "matando" al guaraní es la "castellanización" (de Guarania 2008: 19), esto es, la incorporación indiscriminada de palabras provenientes del español (Cadogan 1958: 23; Galeano Olivera 2004) lo que se debería remediar a través de la enseñanza. Para otros, mientras que los préstamos atestiguan la vitalidad de la lengua (Melià 1992: 185), lo que es preocupante es justamente la discrepancia entre la lengua hablada y los modelos de referencia de la educación (de Granda 1980/81; Corvalán 1981; MEC 2001: 192-196). Esta preocupación surge de la observación de que, a pesar de la función identitaria del guaraní, existen actitudes negativas que los paraguayos atribuyen a sus propias prácticas del habla (de Granda 1980/81:804; Melià 1992: 185), las cuales -dada la larga historia de contacto entre las dos lenguas-se caracterizan por un amplio espectro de fenómenos de convergencia lingüística (de Granda 1996; Kallfell 2010).

Ya en el 1980, en este mismo Boletín, Germán de Granda (1980/81) advirtió acerca de la emergencia de un tipo de diglosia de segundo nivel entre prácticas discursivas cotidianas y la construcción ideológica de un guaraní "puro" por la comunidad de hablantes. Tal constatación la realizó al criticar un programa de alfabetización en guaraní que se estaba elaborando, en ese entonces, usando un "guaraní científico" en la enseñanza, distante de las normas de uso de la lengua, sobre todo porque incorporaba una gran cantidad de neologismos. Durante los últimos 30 años, este programa fue modificado varias veces (Corvalán 1999; Gynan 2001; MEC 2001), pero los debates acerca de la educación bilingüe no han cesado. Y aunque los actores y las alianzas entre ellos cambiaron, el frente principal de batalla sigue siendo entre los defensores de una versión "pura" del guaraní y los partidarios de una versión "híbrida", esto es, una versión que permitiría la inclusión de préstamos del castellano en el discurso guaraní. Esta controversia forma la base del análisis que sigue.

En esta tarea, debo anticipar, mi intención no será defender una posición u otra. Más bien, será mostrar que lo que subyace a ambos proyectos, aparentemente opuestos, son presuposiciones epistemológicas compartidas, las que probablemente estén en la base del fracaso de ellos al enfrentar la compleja realidad sociolingüística del Paraguay. Mi tesis es que, más allá de sostener una postura "purista" e "hibridista" sobre la lengua, respectivamente, ambos proyectos emplean estrategias de purificación, 
aunque diseñadas de manera diferente. Tal análisis permitirá añadir una nueva interpretación a la literatura sobre el bilingüismo paraguayo que, tal vez, contribuya a entender mejor la gran paradoja del guaraní, esto es, al mismo tiempo ser "un objeto de orgullo nacional y uno de estigma social" (Mortimer 2006: 69, traducción propia).

El análisis se basa en datos recolectados en los años 2005 y $2006^{2}$. Desde entonces hubo algunas modificaciones en el programa de educación bilingüe, sin que eso haya apaciguado los ánimos ${ }^{3}$. Los cambios recientes afectan una parte del argumento, por lo que una parte de mi análisis ya no se aplica a la situación actual. Sin embargo, estimo que aquello no resta pertinencia a las conclusiones a las que arribo; al contrario, estos cambios recientes lo que hacen es confirmar mi análisis de la situación, implicancia sobre la que volveré al final del artículo.

El modelo teórico que va a guiar este trabajo es el de "ideologías lingüísticas" ${ }^{4}$, del que presentaré algunos aspectos que van a enmarcar la discusión que sigue. Tras esto, continuaré con unas consideraciones históricas acerca del guaraní y de la educación bilingüe en el Paraguay, para luego concluir con el análisis de la controversia actual sobre su normativización en el sistema educativo 5 .

2 Los datos que recolecté incluyen entrevistas con representantes de órganos gubernamentales e instituciones privadas involucradas en la educación bilingüe y la enseñanza del guaraní, materiales didácticos, y discusiones en los medios y trabajos académicos sobre el tema. La investigación formó parte de mi maestría en antropología. En este ensayo estoy revisitando y expandiendo una parte de mi tesis (Hauck 2009).

3 Pude acompañar el desarrollo de la situación desde cerca por haber continuado trabajando en el Paraguay en otro proyecto, a saber, un proyecto de documentación lingüística de la lengua aché, lengua indígena hablada en la parte oriental del país (ver: http://dobes.mpi. $\mathrm{nl} /$ projects/ache/).

4 Ver la introducción a este volumen para una discusión del concepto de ideología lingüística en relación con otros enfoques. Woolard y Schieffelin (1994), Woolard (1998) y Kroskrity (2010) ofrecen introducciones comprehensivas del tema.

5 Es pertinente esclarecer de antemano que mi interés aquí no es lo que se conoce como las actitudes (socio-)lingüísticas de los hablantes en sí, i.e., el carácter, el estatus y el prestigio que los hablantes atribuyen a diferentes lenguas. Aunque importante también, me voy a limitar en este artículo a otra dimensión de las ideologías lingüísticas, a saber, lo que Kroskrity (1998) define como "ideología lingüística dominante" (ver sección siguiente), el modelo del lenguaje subyacente que es importante para entender los problemas y las controversias alrededor de la educación bilingüe en el Paraguay. Es sobreentendido que ideologías dominantes, a su vez, influencian e informan las actitudes sobre las lenguas. Para el lector interesado específicamente en los detalles de las actitudes de los hablantes refiero a los estudios existentes (Choi 2003; Gynan 1998, 2004; MEC 2001; Mortimer 2006; Solé 1996). 


\section{IDEOLOGÍAS LINGÜÍSTICAS Y EL TRABAJO DE PURIFICACIÓN E HIBRIDACIÓN}

"Una definición del lenguaje siempre es, implícita o explícitamente, una definición de los seres humanos en el mundo" (Williams 1977: 21, traducción propia). Esta declaración de Raymond Williams constituye uno de los supuestos axiomáticos de las investigaciones sobre ideologías lingüísticas, a saber, que las ideas sobre el lenguaje nunca se refieren solamente a éste, sino que, antes bien, ellas vinculan el lenguaje "con la identidad personal y de grupo, con la estética, con la moral y con la epistemología" (Woolard y Schieffelin 1994: 56, traducción propia). Esto es lo que la noción de ideología lingüística trata de captar: la forma en que la estructura y el uso del lenguaje están relacionadas con el contexto socio-cultural.

Siguiendo a Paul Kroskrity (2010), sintetizando varias discusiones existentes (Silverstein 1979; Woolard y Schieffelin 1994; Woolard 1998), concibo ideología lingüística como el conjunto de ideas, creencias y sentimientos sobre el lenguaje y su carácter. El término "lenguaje" se debe entender aquí como un concepto inclusivo, abarcando lenguas, registros, géneros y estilos, fenómenos como code-switching, y también la idea o el concepto de lenguaje en sí mismo. Las ideas sobre unas formas lingüísticas se pueden extender para influir en la percepción y el uso de otras formas, convirtiéndose en ideologías lingüísticas "dominantes" (Kroskrity 1998). Además, lo que plantea el modelo es que suposiciones acerca de cualquier lengua o forma de hablar particular necesariamente dependen de ideas acerca de lo que el lenguaje en general "es"; y el concepto del "lenguaje" a su vez depende de las ideas específicas acerca de las lenguas particulares.

La importancia de las ideologías lingüísticas para entender la naturaleza y complejidad de situaciones de contacto lingüístico, bi- y multilingüismo ha sido reconocida ampliamente (al respecto, ver los artículos en Makihara y Schieffelin 2007; Kroskrity y Field 2009), ya que es en el ámbito ideológico donde se producen las asociaciones de diferentes lenguas con las identidades étnicas y culturales de los hablantes (Kroskrity 1999) y las valorizaciones de estas. Elemental en este contexto es el concepto de indexicalidad, proveniente de la filosofía de Charles S. Peirce (1998 [1893-1913]: 273ff). Con este concepto se busca entender cómo las formas lingüísticas pueden señalar -o constituir un "índice" de- aspectos del contexto socio-cultural, incluyendo los grupos de hablantes (Silverstein 1976; Ochs 1990). De la misma manera que los términos deícticos, los pronombres personales o los marcadores temporales son índices que tienen referentes cambiantes, la pronunciación, la voz, el acento o las variables del estilo pueden ser índices de la proveniencia, 
del género, o de la relación entre los hablantes. Así, diferentes códigos en contextos multilingües señalan/indican, inevitablemente, diferentes aspectos sociales y culturales de los hablantes y grupos de hablantes. Estas asociaciones entre índices lingüísticos y sus referentes socio-culturales son mediadas semióticamente por las ideologías lingüísticas.

Vinculando las lenguas y sus hablantes, las ideologías lingüísticas nunca son ideas neutrales, por lo que toda construcción o definición del lenguaje es una construcción interesada, cargada con los intereses morales y políticos de un grupo social o cultural particular (Irvine 1989: 255; Kroskrity 2010: 195). Es importante señalar, en este sentido, que las ideologías lingüísticas no tienen que ser necesariamente articuladas de manera explícita, sino que pueden operar también por debajo de la conciencia de los hablantes. Al respecto, Kroskrity (1998) recurre a la distinción entre conciencia práctica y conciencia discursiva de Anthony Giddens (1979) para pensar los diferentes grados de conciencia que los hablantes pueden tener de las ideologías lingüísticas. Giddens (1979: 6) relaciona la conciencia discursiva que los actores tienen de los sistemas sociales en los que participan con el grado de control sobre dichos sistemas. De esta manera, cuanto más tengan los actores conciencia discursiva de las estructuras dominantes, más control podrán ejercer en el desarrollo de los eventos ${ }^{6}$. Siguiendo este razonamiento, Kroskrity (2010: 198, traducción propia) sugiere que altos niveles de conciencia discursiva están correlacionados con "contestación activa de ideologías lingüísticas", y por el contrario, la conciencia práctica está correlacionada con "ideologías relativamente indiscutibles, altamente naturalizados, y definitivamente dominantes".

En lo que sigue me dedicaré a un complejo ideológico en particular, rotulado como "ideología de lengua estándar" (cf. Milroy y Milroy 1985; Lippi-Green 1997), y, específicamente, de un estándar concebido como homogéneo y "puro". Este complejo se ha consolidado como el modelo hegemónico del lenguaje en las sociedades occidentales y se ha impuesto también en el Paraguay, tal como mostraré más adelante. A modo de definición, Lippi-Green (1997: 64, traducción propia) caracteriza la ideología de lengua estándar como una "tendencia hacia un lenguaje abstracto, idealizado y homogéneo, que es impuesto y mantenido por las instituciones dominantes y que designa el lenguaje escrito como su modelo, pero que ha sido elaborado, en primer lugar, a partir del habla de la clase media

6 Argumentos paralelos de la teoría social se encuentran en los trabajos de Williams (1977) y Bourdieu (1985 [1982]). 
alta". A su vez, por "purismo lingüístico", siguiendo a Makihara (2007: 50, traducción propia), se entenderá la "insistencia en la pureza o corrección de formas lingüísticas", evitando la influencia de otras lenguas. Generalmente el purismo se impone por las instituciones que controlan la estandarización de las lenguas, como academias lingüísticas o instituciones de enseñanza.

Como ha demostrado Michael Silverstein (1996: 285, traducción propia), en la medida en que las prácticas lingüísticas estandarizadas alcanzan una "hegemonía explícitamente reconocida sobre la definición de las normas de la comunidad", otros usos lingüísticos llegan a ser evaluados también con referencia a este estándar (1996: 287). Así, formas sincréticas del habla y usos heterogéneos del lenguaje son evaluados negativamente por su disconformidad con el estándar. Es esta ideología la que es responsable, en gran parte, de la jerarquización entre formas lingüísticas subyacente a las situaciones de diglosia.

El análisis más comprehensivo del advenimiento de esta ideología, y de las políticas de desigualdad incorporadas en ella, fue hecho por Richard Bauman y Charles Briggs (2003) en su libro Voices of Modernity. En esta obra se basan en el argumento de Bruno Latour (2007 [1991]), quien propone que la llamada modernidad no nace del surgimiento de las ciencias o del pensar científico per se, sino mas bien de la producción de un "gran divisor" entre el mundo natural y el social -un proceso que este llama el "trabajo de purificación"- junto a su constante mediación y traducción -el "trabajo de hibridación"-. Bauman y Briggs, por su parte, expanden este argumento planteando que el gran divisor no se produjo solo entre los reinos de la naturaleza y de la sociedad, sino que además esta purificación fue de la mano de la construcción del lenguaje como dominio autónomo en beneficio de la representabilidad científica y política transparente.

Filósofos como John Locke y otros convirtieron el lenguaje en un medio de representación preciso, neutral, depurado de todas las referencias a su uso por gente inculta en su vida cotidiana, tal y como lo exigían las nuevas ciencias de la naturaleza y las nuevas formas de organización política. Fue este modelo del lenguaje el que sirvió como matriz para la creación de las lenguas estándar de los Estados-nación emergentes. Pero así como la purificación de naturaleza y sociedad era vulnerable a la "proliferación de híbridos" por medio del inevitable trabajo de traducción (Latour 2007 [1991]), la purificación del lenguaje era vulnerable a la hibridación por medio de su función indexical. Esto es, aunque desvinculadas en teoría de sus conexiones con un cierto lugar, ciertas personas y sus condiciones de producción, las lenguas estándar, supuestamente "neutrales" y "desinteresadas", en la práctica se transformaron en los índices del estatus social de sus creadores: la élite intelectual. Al mismo tiempo, las maneras del habla no-estándar 
fueron transformadas "en marcadores de irracionalidad, ignorancia, fracaso escolar e idoneidad para trabajos de préstamo de servicios sin porvenir" (Bauman y Briggs 2003: 7, traducción propia), índices de los pobres, las mujeres, la clase trabajadora, y los no-europeos. Hablar la modalidad estándar garantizaba la participación libre en la esfera pública de la sociedad y, por tanto, era la práctica que se esperaba de todos los sujetos. Así, la habilidad de subordinarse a prácticas discursivas específicas determinaba el acceso a la esfera pública y el nivel en el que uno estaba habilitado a "hablar", en el sentido que le da Gayatri Spivak (1988). De esta forma, las lenguas estándar fueron convertidas en instrumentos poderosos para controlar la inmigración y movilidad social hacia arriba (Bourdieu 1985 [1982]).

Distinguimos aquí dos niveles de purificación/hibridación entonces: uno es la construcción ("purificación") del lenguaje como lenguaje y su concomitante e inevitable vulnerabilidad a la hibridación indexical; y el otro es la purificación de una lengua (cualquiera) y la concomitante construcción de prácticas lingüísticas impuras o híbridas. Entonces, construcciones lingüísticas e indexicalidades particulares son el producto de una concepción del lenguaje de fondo, que llegó a ser la ideología lingüística de "estándar monoglósico" (Silverstein 1996) en los Estados-nación modernos y también el modelo científico del lenguaje para las investigaciones lingüísticas.

Proliferaron las críticas a este paradigma, ejemplificado en los trabajos de Ferdinand de Saussure y sus seguidores (Derrida 1971 [1967]; Deleuze y Guattari 1994 [1980]; Bourdieu 1985 [1982]). Según Bourdieu (1990), el paradigma sufre de lo que el llama la "falacia escolástica": El proyecto de de Saussure (1945 [1916]) pretendía abandonar el principio de representación para su modelo semiótico. Concibiéndolo como mecanismo de articulación entre términos cuyo significado reside en la diferencia con otros términos, surge el lenguaje (langue) como objeto independiente del mundo real y de su uso (parole). Sin embargo, al mismo tiempo que el lenguaje llega a ser una entidad inteligible, aunque esta ya no representa un mundo preo extra-lingüístico, una relación representacional surge de nuevo como analogía estructural entre el sistema de lenguaje universal y las prácticas del habla cotidianas, heterogéneas e híbridas, de las cuales es supuestamente el modelo (Krämer 2001: 104-105). Así, el habla inevitablemente se torna en una representación del primero. En la terminología de Clifford Geertz (1973 [1966]: 93), el modelo de la realidad llega a ser el modelo para la realidad, lo que, obviamente, resulta en una desventaja para la realidad, pues el habla, por definición siempre será algo incompleto, imperfecto y deficiente. Y tanto como era por medio de la escrita que de Saussure pudo demostrar la autonomía de la langue, es con referencia a esta que se mide la divergencia del habla. 
Para entender la paradójica existencia de este complejo ideológico en un país tan heterogéneo lingüísticamente como el Paraguay, lo relacionaré con otro concepto que va a completar el repertorio teórico con el que podemos analizar esta situación: lo que Jean y John Comaroff (1991), a partir de su trabajo en Sudáfrica, llaman la "colonización de la conciencia", esto es, los efectos que la descalificación y desautorización de epistemologías indígenas -que suele acompañar a las políticas coloniales e imperiales- tienen en los sujetos coloniales y poscoloniales; y, también, en tanto modo en que la resistencia local está moldeada por los discursos hegemónicos importados (ver Mignolo 2000). En relación con la resistencia lingüística en contra de lenguas hegemónicas, se ha observado en muchas regiones del mundo que el activismo a favor de lenguas minoritarias o marginalizadas muchas veces depende de y reproduce las ideologías dominantes del lenguaje que formaban parte de las políticas que llevaron a la opresión de las lenguas en cuestión (Woolard 1998: 17). Especialmente, las ideas de purismo lingüístico - una parte esencial de la retórica de mantenimiento lingüístico- muchas veces se asemejan a las de las ideologías de estándar monoglósico occidentales, basadas en modelos logocéntricos del lenguaje (Derrida 1971 [1967]; Whiteley 2003). Estos modelos, muchas veces, se oponen a ideologías de tradiciones orales que se destacan por su énfasis de la diversidad, la variabilidad, la contextualidad y la performance (Bauman y Briggs 1990), pudiendo crear una brecha entre el uso cotidiano de la lengua en cuestión y una variedad oficial purificada, perjudicando, sin pretenderlo, el uso de esta y llevando al conflicto generacional y a la resistencia al mantenimiento lingüístico.

\section{EL GUARANÍ EN PARAGUAY: BILINGÜISMO CON DIGLOSIA}

Shaw Gynan (2001: 78, traducción propia) atribuye una buena parte "del mérito (o de la culpa) de la supervivencia del guaraní" al sistema de la encomienda y a los misioneros franciscanos y jesuitas entre los siglos XVI a XVIII. La encomienda era una institución mediante la cual se concedía a un colonizador un grupo de indígenas que debían trabajar y pagar tributos para el encomendero. En el marco de este sistema, el concubinato con mujeres guaraníes y la poligamia, alentados por los gobernadores, fueron factores decisivos para el mantenimiento del guaraní, puesto que los hijos aprendían guaraní de sus madres (Gynan 2001: 79; Melià 1992). Al 
mismo tiempo, en oposición a la encomienda, los misioneros franciscanos y, después, jesuitas se empeñaron en el proselitismo en la lengua guaraní en las famosas "reducciones", los pueblos misionales (Melià 1986, 1992). Eso no solo contribuyó al mantenimiento del guaraní sino también a lo que Bartomeu Melià (1986) llama la "conquista y reducción" del guaraní, i.e., su estandarización y textualización.

La posición del guaraní como el idioma principal de Paraguay se vio consolidada después de la independencia en el 1811. En el conflicto con Argentina, que no quería reconocer la secesión de Paraguay, los gobernadores dependieron del apoyo de la población guaraní-hablante, al punto que el dictador José Gaspar Rodríguez de Francia decretara que los europeos y criollos deberían casarse con mujeres de ascendencia guaraní (Potthast 1999: 350-352; Gynan 2001: 81-82). En las guerras de la Triple Alianza (1864-1870) y del Chaco (1932-1935), la lengua fue usada "como santo y seña en las batallas, se publicaron periódicos en guaraní para ser distribuidos entre los soldados, cantos y música sirvieron de entretenimiento al ejército y las arengas fueron en guaraní" (Corvalán 1981: 180; Lustig 1999). Así, el guaraní se convirtió en la lengua de la liberación nacional y un ícono de la identidad paraguaya.

Sin embargo, y a pesar de este significado histórico, la política hacia el guaraní siempre ha sido ambigua, pues, aunque fuese considerado característica cultural importante, su empleo quedó limitado al uso oral y, principalmente, a la literatura de carácter folklórico, tal como lo expresa Lustig (1999: 363): "Desde finales del siglo XIX la lengua guaraní se ha transformado en un símbolo de la identidad paraguaya -sin que esto haya favorecido mucho su oficialización o su uso real como medio de comunicación en los ámbitos reservados tradicionalmente al español". La explicación a esta ambivalencia en la consideración del guaraní puede encontrarse en el histórico patrocinio de la élite criolla gobernante a las poblaciones rurales guaraní-hablantes a cambio de su lealtad política, sin que la élite comenzase a utilizar la lengua guaraní (Gynan 2001: 85). Esto se volvió más explícito en el tiempo de la guerra del Chaco, cuando el guaraní fue prohibido en la escuela mientras que el castellano fue prohibido entre los soldados (Steckbauer 1999: 384-385). La elevación del guaraní al estatus de lengua nacional por el dictador Alfredo Stroessner en el 1967 también debe ser entendida en este contexto (Melià 1992: 173). La única lengua oficial era y continuaba siendo el español, ya que la alfabetización y la educación eran solamente en castellano. Es a partir de este derrotero histórico que debemos interpretar la descripción usual de la situación del Paraguay como de "bilingüismo con diglosia" (Fishman 1967). Aunque los guaraní-hablantes 
son la mayoría de la población ${ }^{7}$, y no obstante la nueva constitución posdictadura estableció igualdad lingüística de iure, el castellano sigue siendo la lengua dominante de facto (Gynan 2001). El guaraní, aunque sea valorado positivamente como emblema nacional, es a la vez un índice de bajo nivel educacional, ignorancia, subdesarrollo y ausencia de progreso.

Esta ambigüedad y aparente carácter contradictorio entre las representaciones sociales acerca del guaraní se explican por la coexistencia de diferentes ideologías lingüísticas, aspecto que no debe causar extrañeza, toda vez que los teóricos del modelo entienden el carácter múltiple de las ideologías lingüísticas en tanto cada comunidad también presenta una pluralidad de divisiones sociales (Kroskrity 2010: 197). Y esto es lo que se observa en Paraguay: la asimetría entre las dos lenguas -guaraní y castellano- refleja la asimetría entre clases sociales y localidades, pero, al mismo tiempo, el guaraní distingue el Paraguay de los países vecinos por lo que es un símbolo positivo para la representación de la identidad nacional. Esta ambigüedad de su función indexical garantiza que los debates sobre el guaraní no cedan. Frente a este panorama, con el fin de remediar su estatus inferior, varias propuestas para la inclusión del guaraní en el sistema educativo han surgido durante los últimos 30 años. En la siguiente sección revisaré el desarrollo de la educación bilingüe en Paraguay, para así analizar posteriormente las controversias actuales en torno a esta situación.

\section{LA EDUCACIÓN BILINGÜE}

A pesar de que la mayoría de los alumnos en el Paraguay tenían el guaraní como lengua materna, no se le usó en la enseñanza hasta el año 1983; incluso hubo tiempos donde su uso en la escuela fue prohibido completamente (Gynan 2001: 93; MEC 2001). Las críticas al escaso rendimiento escolar y al alto nivel de deserción escolar (Corvalán 1981; de Granda 1980/81) finalmente llevaron a la incorporación del guaraní al sistema educativo en el 1983 (Corvalán 1999: 20). De manera heurística, siguiendo lo que establece Gynan (2001), distinguiré tres fases en la educación bilingüe en el Paraguay: el modelo de transición (1983-1992), el modelo de mantenimiento (1994-

7 El 29\% de la población fue clasificada como guaraní-hablante, el 53\% como bilingüe y el $10 \%$ como hispano-hablante (Gynan 2007). 
1999) y la fase actual (1999-presente). Discutiré las primeras dos aquí, reservando la fase actual y sus controversias para la sección final.

El modelo de transición estaba orientado al cambio progresivo hacia el castellano usando el guaraní para facilitar el aprendizaje de éste (MEC 2006: 8-9). Toda vez que la meta era castellanizar a la población monolingüe guaraní, lo que se hacía era continuar la política anterior de "castellanización directa" y perpetuar el estado de diglosia (Melià 1986: 264). Sin embargo, al evaluarlo históricamente, aun con respecto a la competencia en castellano los resultados fueron claramente negativos y el programa "resultó en un mayor nivel de repitencia y deserción, y sobre todo en un bajísimo nivel de rendimiento educativo" (Corvalán 1999: 20).

Solo después de la caída de la dictadura de Stroessner, en 1989, el modelo anterior fue reemplazado por el modelo de mantenimiento, implementado con el Plan Nacional de Educación Bilingüe en 1994 (Comisión Nacional de Bilingüismo 1997), teniendo como meta lograr un "sistema bidireccional completamente equilibrado" (Gynan 2001: 87; MEC 2006). El modelo reflejaba la nueva constitución posdictadura de 1992, que definía ambos idiomas como oficiales en igualdad de condiciones (Paraguay 1992). Planteaba que, al entrar en la escuela, los educandos aprenderían a leer y escribir primero en su lengua materna, sea ésta el guaraní (sobre todo en áreas rurales) o el castellano (en las ciudades), y su respectiva segunda lengua sería usada para la enseñanza, progresivamente, hasta un equilibrio del 50 por ciento en el noveno grado (Comisión Nacional de Bilingüismo 1997; MEC 2006). Aunque el programa fue bienintencionado, los resultados distaron mucho de ser satisfactorios (Gynan 2001: 76), siendo la ausencia de recursos y la falta de experiencia algunos factores para su fracaso. Sin embargo, la razón principal fue que la población se mostró reacia a su implementación (MEC 2001). Este rechazo fue causado, por un lado, porque el guaraní no tenía utilidad para la movilidad social que la población guaraní-hablante sí le atribuía al castellano (al ser la lengua de la élite y una lengua internacional), y, por otro lado, porque la versión del guaraní que se usó distaba de las normas del uso de esta lengua. Respecto a esto último, dado que el programa fue diseñado para producir hablantes bilingües coordinados que pudieran usar el guaraní y el castellano en cualquier situación con habilidad semejante, "manteniendo los códigos de los sistemas separados" (MEC citado en Gynan 2001: 97), la formación de palabras estaba restringida a raíces guaraní, lo que causó la emergencia de una cantidad considerable de neologismos con los que los hablantes no tenían familiaridad (MEC 2001: 192). Así, finalmente, aunque se le atribuía alto prestigio a esta versión del guaraní, que se asociaba con el guaraniete, el "guaraní real" tradicional, ella presentó un obstáculo para alcanzar a las metas educativas (MEC 2001, 2006). 
Como he sugerido arriba, la realidad sociolingüística de Paraguay es mucho más compleja que los términos bilingüismo o diglosia sugieren. El llamado guaraniete, el guaraní tradicional puro, es más un símbolo de referencia que una lengua que realmente se escucha en el discurso cotidiano, y existen opiniones divergentes sobre su forma real (de Granda 1988; Lustig 1999; Melià 1986, 1992). Sin embargo, todos están de acuerdo en que lo que se habla en Paraguay hoy en día no lo es. El discurso cotidiano se caracteriza por un amplio espectro de fenómenos de contacto lingüístico como el codeswitching, el code-mixing y la convergencia lingüística (de Granda 1988, 1996). El término que se usa para esta lengua es jopara, la palabra guaraní que designa una mezcla o un conjunto de cosas diferentes. Melià (1992: 184) escribe: "No es tarea fácil definir qué tipo de lengua es ese jopara, ya que la falta de normatividad es tan marcada que desafía cualquier categorización".

Hay muchos estudios que muestran que la mayoría de situaciones donde ocurre code-switching o -mixing, no están caracterizadas por una "falta de normatividad". El cómo, el cuándo y el dónde cambiar los códigos sigue esquemas claros y los valores simbólicos que se atribuyen a una lengua o a un código, y al hecho de cambiar en sí mismo, son importantes (Woolard 2004; Auer 1998). En Paraguay eso también es así (de Granda 1988, 1996; Kallfell 2010). No obstante, el punto importante es que la idea de una "falta de normatividad" existe y es eso que lleva a la gente a condenar su propia forma de hablar ${ }^{8}$.

"Hablamos mal el guaraní" se escucha frecuentemente. Tal como se ha relatado en referencia a otras situaciones donde el contacto prolongado entre dos lenguas llevó a prácticas discursivas sincréticas (Hill y Hill 1986), aunque se ve natural cambiar entre los dos idiomas, la gente menosprecia "la mezcla" como uso de lenguaje incorrecto e imperfecto (Melià 1992: 185; Mortimer 2006: 60) ${ }^{9}$. Esta es otra dimensión de la paradoja del Paraguay: la valorización positiva que el guaraní recibe como símbolo nacional contrasta específicamente con las actitudes negativas que los paraguayos tienen con respecto a sus prácticas del habla cotidiano. Entonces, a pesar de que el imaginario nacional asocie el guaraní con la resistencia a la asimilación cultural, no parece haber podido resistirse a una cierta asimilación lingüísticaideológica. Aun en un país bilingüe como Paraguay, la opinión popular sobre las lenguas está dominada por la ideología del estándar monoglósico.

\footnotetext{
8 No todos los estudiosos del bilingüismo paraguayo comparten esta visión pesimista (cf. Gynan 2001, 2004).

9 Es más que eso: “ya no hablamos bien el guaraní” (citado en Melià 1992: 185, énfasis en el original) indica un ideal perdido de alguna versión original del guaraní.
} 
Las muchas palabras incorporadas al guaraní desde los tiempos jesuitas atestiguan que tal ideología es diametralmente opuesta a una ideología tradicional "sincrética" (Kroskrity 2009), aunque sea con referencia a la primera que las prácticas discursivas cotidianas sean evaluadas hoy en día. Por esta razón es que de Granda (1980/81) entregaba argumentos en contra de la incorporación de un guaraní "puro" en la enseñanza, en tanto aquello profundizaría las actitudes negativas y reproduciría recursivamente una diglosia entre estas y el guaraní académico ${ }^{10}$. Investigaciones en varios contextos han mostrado que construir la pureza de una lengua vernácula puede perjudicar la misma, por medio de crear un abismo entre el uso cotidiano de ella y una variedad oficial purificada que se enseña en la escuela. Esto puede llevar a la "inseguridad lingüística" (Labov 1966) de los hablantes y así incitar el desplazamiento lingüístico (ver Kroskrity y Field 2009). En una medida controversial, planificadores lingüísticos del Ministerio de Educación y Cultura han intentado responder a este desafío, acreditando una versión nueva "híbrida" del guaraní para el uso en las escuelas. Al análisis de esta versión me dedicaré en la sección final.

\section{FONOLOGÍA, ORTOGRAFÍA Y LA MODERNIZACIÓN DE LA EDUCACIÓN BILINGÜE}

En 1999, el Ministerio de Educación y Cultura del Paraguay (MEC) reformuló el programa de educación bilingüe considerando dos dimensiones: (a) su flexibilización con relación a la distribución y nivelación de las dos lenguas (MEC 2001: 195); (b) la versión del guaraní que se usaría en la enseñanza. Sobre esto último, respondiendo a las críticas y dando cuenta de las prácticas del habla cotidianas, el MEC permitió la inclusión de términos castellanos en el discurso guaraní en tanto fueran integrados a la estructura guaraní. El término que el MEC usa para estos préstamos es jehe'a, otra palabra guaraní para "mezclar" (cf. Boidin 2000). Según la definición del MEC el jehe'a, en oposición al jopara, es el "préstamo lexicológico integrado

10 De Granda (1980/81: 804) usa el término "doble diglosia interna" con lo que se refiere "no a la existente, según algunos, en la relación español-guaraní y sí a la que podría generarse entre variantes altas y bajas de cada una de estas lenguas, con las graves consecuencias que de ello se derivarían en los ámbitos lingüístico y sociocultural e, incluso, en el político". Aquí me limito a comentar sobre la situación del guaraní. 
a la estructura fonomorfosintáctica del guaraní. Al integrarse a esta estructura ya forma parte, de hecho, del corpus lexical del guaraní y cumple con las normativas ortográficas de esta lengua" (MEC 2006: 68). Jopara lo define, en cambio, como "préstamo lexicológico no integrado a la estructura fonológica y/o morfosintáctica del guaraní” (2006: 68). Por sinécdoque jehe’a llegó a designar la variedad del guaraní en sí, que permite estos préstamos.

Los siguientes son algunos ejemplos de materiales didácticos publicados por el MEC (morfemas provenientes del castellano en negrita) ${ }^{11}$ :

Amongurusu kuádrope ...

A-mo-kurusu kuádro-pe

1S-CAUS-cruZ cuadro-LOC

Marcá con X en los cuadros ...

(2)

Arrestakuaaitereímaningo.

A-rresta-kuaa-itereí-ma-ningo

1S-restar-know-mucho-PFV-EMPH

Ya sé muy bien como restar.

(3)

Edivuha ha ekopia letrakuéra ne kuaderno-pe.

\begin{tabular}{|c|c|c|}
\hline E-divuha & ha e-kopia & letra-kuéra ne \\
\hline 2S.IMP-dibujar y & 2S.IMP-copiar & letra-PL $2 S$ \\
\hline
\end{tabular}

(4)

Ajesareko ha aipapa [dibujito de pelota] oĩva kartónpe.

A-jesareko ha ai-papa [dibujito de pelota] oĩ-va kartón-pe

$1 \mathrm{~S}-$ atender y $1 \mathrm{~S}-\mathrm{contar}---\quad$ hay-NOM cartón-LOC

Observo y cuento las [dibujito de pelota] que hay en cada cartón.

11 Leyenda: línea 1: texto original; línea 2: morfemas (negrita indica morfemas prestados del castellano); línea 3: glosa interlinear; línea 4: traducción libre. CAUS: causativo; COMP: comparativo; COMPL: completivo; EMPH: marcador enfático; FUT: futuro; IMP: imperativo; LOC: locativo; NOM: nominalizador; PFV: perfectivo; 1,2,3 persona; S: singular; PL: plural. - Fuente: Ejemplo (1) de Medio Natural y Salud. Ñañangareko kuaa hagua ñandú rekoha ha ñande reta rehe. $1^{\circ}$ grado. E.E.B. 2009; ejemplos (2) y (4) de Matemática. Nandekatupyry hagua papapykuérape. $1^{\circ}$ grado. E.E.B. 2009; ejemplos (3), (5) y (6) de Péicha Ñañepyrũ Trazando un Camino; todos publicados por el Ministerio de Educación y Cultura del Paraguay, Asunción. 
(5)

Eskrivimi ne kuaderno-pe mbohapy kósa ndegustavéva ...

Eskrivi-mi ne kuaderno-pe mbohapy kósa nde-gusta-vé-va

Escribir-POL 2S cuaderno-LOC tres cosa 2S-gustar-COMP-NOM

Por favor, escribí en tu cuaderno las tres cosas que más te gusten ...

(6)

Ko árape reikuaáta tékstopa guaraníme ha avei kastellánope.

Ko ára-pe rei-kuaá-ta téksto-pa guaraní-me ha avei kastelláno-pe este día-LOC $2 \mathrm{~S}$-saber-FuT texto-COMPL guaraní-LOC y también castellano-LOC Hoy vas a aprender un texto entero en guaraní y en castellano.

Los materiales muestran claramente la intención del MEC de adaptar el guaraní de la enseñanza a la forma del habla que se usa en el día a día. Sin embargo, no tuvieron una buena acogida. Entre las críticas que se suscitaron, muchas seguían el esquema de ideologías lingüísticas puristas, que ven en el jehe 'a la corrupción y degeneración del guaraní ${ }^{12}$. Ya exploré los peligros inherentes en estas ideologías en la sección anterior. Pero hay otras críticas más interesantes de evaluar en tanto señalan los problemas subyacentes de la propuesta. Para presentar este análisis, previamente debemos revisar algunos aspectos de la fonología del guaraní.

La estructura silábica canónica del guaraní es $(C) \mathrm{V}(\mathrm{V})$. No existen las sílabas que terminan en consonantes o grupos consonánticos con excepción de oclusivas prenasalizadas sonoras $\left[{ }^{\mathrm{m} b}\right]<\mathrm{mb}>$ or $\left[{ }^{\mathrm{n}} \mathrm{d}\right]<\mathrm{nd}>$ y la secuencia $\left[{ }^{\mathrm{n}} \mathrm{t}\right]<\mathrm{nt}>$. Las palabras jehe' $a$ provenientes del castellano en la forma en que están representadas en los ejemplos arriba no respetan esta regla. Escritos en ortografía guaraní, pero con la intención de reproducir su forma original, presentan una dificultad para el hablante nativo. La palabra $<$ téksto $>$ por ejemplo tiene la estructura 'CVCC.CV. Esta no es natural para el hablante nativo, ya que incluye una secuencia de tres consonantes, k-s-t. Lo mismo ocurre con la palabra <eksponénte $>$, VCC.CV.'CVC.CV. Uno de los críticos observa:

[L]o máximo que un Guarani-hablante podrá decir es ey-po-né-nte, pero nunca eksponénte. Por esta misma razón, es incorrecta la nueva palabra

12 Uno de los críticos escribe: “[L]os jehe’a del Guarani y del castellano no son otra cosa que la fiel demostración de nuestra pereza intelectual y de nuestro conformismo social. ... En el fondo, el intento -hoy- de aceptar el jehe'a en la educación no es otra cosa que la alegre aceptación de nuestra mediocridad lingüística e intelectual" (Galeano Olivera 2004, énfasis omitido). 
"provléma" ... en lugar de "provléma", el paraguayo dirá "poléma (polé-ma)" (Galeano Olivera 2004).

"Ndaipori pauléma", "no hay problema", es un dicho que se escucha frecuentemente en Paraguay, evidencia de que sí se hace amplio uso de préstamos del castellano en el discurso guaraní. Pero estos préstamos se incorporan justamente no respetando la estructura fonológica de la lengua base. Eso fue, probablemente, el esquema que dominó el contacto lingüístico desde tiempos jesuíticos (Melià 1986). El léxico guaraní incluye una gran cantidad de ítems provenientes del castellano, como vaka, kavaju, kesu (vaca, caballo, queso, respectivamente), que atestiguan la actitud receptiva del guaraní a la incorporación de elementos de otra lengua, pero siempre adaptándolos a su estructura fonológica ${ }^{13}$. Particularmente, no se toleran grupos consonánticos, tal como lo evidencian las palabras kurusu (cruz) o kavara (cabra), que fueron adaptadas por medio de epéntesis vocálica. Más aun, no solo los préstamos son adaptados a la fonología del guaraní, sino también aspectos fonéticos de la variedad de castellano hablada en Paraguay. Como demuestran estudios de esta (de Granda 1988: 92; Penner et al. 2009: 409), los paraguayos tienden a pronunciar el primer segmento de grupos consonánticos en general como la vocal central cerrada [ $\square]$, representada en la ortografía guaraní como $\langle\mathrm{y}\rangle$. Las secuencias $<$ sk $>,<\mathrm{kt}\rangle,\langle\mathrm{ks}\rangle$, o $\langle$ st $>$ por ejemplo: oscuridad se pronuncia como oycuridad; excelente como eyselente $^{14}$.

Considerando este panorama, podríamos hablar de dos tipos de ideologías lingüísticas dominantes tradicionales: una ideología sincrética, que favorece la incorporación de elementos léxicos, y una ideología de conservación fonológica del guaraní, que impacta la realización fonética de préstamos castellanos y también el discurso castellano en general. Volviendo a los materiales escolares, estas observaciones demuestran, en primer lugar, que los parámetros del Ministerio de Educación y Cultura no son consistentes, pues las nuevas palabras no se integraron en la "estructura fonomorfosintáctica" del guaraní como la definición del jehe'a pretende. Simplemente se escriben como si fueran palabras guaraní, lo que muestra que se confundió la fonología con la ortografía. Así, mientras que siguen

13 El contacto lingüístico entre lenguas amerindias en la época precolonial es un tema que sigue siendo insuficientemente explorado, también por ideologías lingüísticas profesionales que toman lenguas homogéneas como su base.

14 Eso es solo un ejemplo de la influencia del guaraní sobre el castellano (cf. Penner et al. 2009). 
la ideología sincrética, incorporando préstamos, las palabras jehe'a van en contra de la ideología lingüística de conservación fonológica, ya que es justamente la incorporación de los préstamos a la estructura fonológica del guaraní la que distingue la forma particular del sincretismo lingüístico paraguayo. Es eso lo que los nuevos materiales didácticos no logran. De esta manera, en vez de ser una forma más cercana a las normas de uso de la lengua, el nuevo guaraní jehe'a otra vez define un estándar que se aleja del discurso cotidiano. Materiales anteriores que usaron el guaraniete, el guaraní "puro", tradicional, fueron criticados por la creación de neologismos, pues restringieron la formación de palabras a raíces guaraníes. Estaban basados en una definición de una lengua estándar que toma como parámetro el léxico, manteniéndolo libre de influencias castellanas. El jehe'a, aunque permitiendo la inclusión de préstamos, en realidad no es un "híbrido", sino otra forma de un estándar puro y homogéneo, solo que en este caso se define a través de la ortografía.

\section{CONCLUSIÓN}

En virtud del análisis expuesto, no solo el guaraniete (el guaraní purificado de préstamos) es un guaraní "puro" entonces. El jehe'a también, aunque diseñado para ser una versión similar a la lengua hablada, híbrida, debido a una confusión de la ortografía con la estructura fonológica, se convirtió en una versión "pura" más. Ambas versiones nacen de una ideología lingüística dominante de estándar monoglósico que subyace a la planificación lingüística en Paraguay. Esta ideología se opone a ideologías de sincretismo lexical y de conservación fonológica que han influenciado el desarrollo de la lengua guaraní a lo largo de su historia de contacto con el castellano. En contra de los defensores del guaraniete, el guaraní siempre ha estado abierto a préstamos del castellano, pero no manteniendo la estructura fonológica de estos, como proponen los partidarios del jehe'a. Así, las dos versiones son invenciones artificiales, que no se parecen a las lenguas habladas por el pueblo paraguayo. No sorprende, por tanto, que ambas fueran rechazadas.

Sin embargo, dependiendo del punto de vista, tanto el guaraniete como el jehe'a también son versiones "híbridas", porque incorporan palabras nuevas, neologismos en un caso, hispanismos en el otro. Bauman y Briggs (2003: 5 , traducción propia) plantean que "la pureza clasificatoria en sí misma es una construcción epistemológica, y cada forma 'pura' también puede ser concebida como híbrida de una manera u otra". En vez de clasificarlas 
como pura o híbrida, más bien podemos identificar en ambos proyectos procesos de purificación y procesos de hibridación al mismo tiempo. Siguiendo el análisis de la producción de la modernidad propuesto por Latour (2007 [1991]) y expandido por Bauman y Briggs (2003) para incluir la construcción "moderna" del lenguaje, podemos observar en Paraguay los mismos mecanismos de producción y purificación de las lenguas estándar, aptas para (y adaptadas a) la inclusión en el sistema educativo, que, a su vez, transforman las maneras del habla no-estándar en índices de irracionalidad e ignorancia. Por medio del trabajo de purificación ideológica, las versiones puras del guaraní y del castellano producen la "impureza" de la forma de hablar cotidiana de la gente. El jopara no es solamente concebido, sino más bien construido como híbrido, al mismo tiempo que se define lo que es el estándar correcto en los libros escolares, sea este castellano, guaraniete o jehe'a. Como vimos arriba, en oposición a este último, que incorpora los préstamos de manera supuestamente ordenada y organizada, el jopara es definido por el MEC como "préstamo lexicológico no integrado" (2006: 68, cursivas son mías) a la estructura fonológica del guaraní.

El jehe'a es un ejemplo perfecto del proceso por el cual el habla, que se cree representada en la lengua escrita según de Saussure y la lingüística moderna, se convierte en una representación del modelo escrito. Éste fue construido supuestamente representando la forma en que las personas hablan, pero tan pronto como ha sido estandarizado, normativizado, e incorporado en el sistema de educación a través de los materiales didácticos, se convierte en el modelo a ser representado por el lenguaje cotidiano. De esta manera, del modelo de la realidad se transforma en el modelo para la realidad. Así, el ideal escrito produce y enfatiza la diferencia de la forma del habla de las personas. Esto no solo es una falacia escolástica, como diría Bourdieu (1990), sino política lingüística. Además, dado el vínculo indexical entre "guaraní" e identidad nacional, construyendo el habla cotidiano (jopara) por su diferencia con el guaraní (guaraniete o jehe ’a), se establece una relación negativa con la representación de esta.

Tras estas propuestas está la idea del guaraní y castellano como estándares monoglósicos homogéneos que se emplea como un instrumento de purificación del habla. Y más que eso: en un clima intelectual cada vez menos favorable a la planificación lingüística purista, la pureza simplemente se transfiere a otro nivel. En el jehe'a ya no se está midiendo la pureza por medio del léxico, ya que han sido permitidas palabras castellanas, sino que por la ortografía, que pasa a ser el nuevo parámetro utilizado para garantizar la existencia purificada de un estándar lingüístico. Por ello, la nueva versión parece ser solo un componente más en los mecanismos que contribuyen al mantenimiento de las fronteras que separan los que hablan y escriben 
correctamente de los que no. El acceso a la esfera pública es así regulado por la capacidad de subordinarse a las prácticas del habla estandarizadas.

La vigencia del jehe'a, tal como he descrito arriba, fue relativamente corta. Se revisaron los materiales y se realizaron nuevos estudios que llevaron al MEC a cambiar su política nuevamente en 2008 y 2009. Como resultado de esto, aunque se siguen usando préstamos en los materiales, ya no se escriben en la ortografía guaraní y se usa la ortografía de la lengua base, el castellano. Es demasiado temprano para hacer predicciones sobre la eficiencia o el fracaso de tales materiales, por lo que debemos estar pendientes a próximos cambios para futuras revisiones. Sin embargo, el hecho de que el mismo MEC abandonara la enseñanza del jehe'a otra vez, resulta en una contundente confirmación del análisis presentado.

La planificación lingüística en Paraguay puede tener como objetivo pretendido fortalecer el guaraní y lograr la equidad funcional entre las dos lenguas oficiales. Sin embargo, las formas en que las lenguas de enseñanza están diseñadas resultan contraproducentes para este objetivo (basta pensar en la confusión de un niño que tiene que aprender las mismas palabras dos veces con diferentes ortografías). Basadas en una ideología lingüística de estándar monoglósico, camuflada en Paraguay por la retórica de bilingüismo, en vez de combatir la situación de diglosia y contribuir a una revalorización del guaraní reinvirtiéndolo con el capital simbólico del cual ha sido privado, ambas versiones amplían la brecha entre las prácticas lingüísticas cotidianas y las habilidades lingüísticas necesarias para la representación de la identidad nacional. Así, alimentan las actitudes negativas de las personas en contra del jopara, irregular, imperfecto, e imposible de clasificar. No es de extrañar entonces que la gente opine "hablamos mal". De esta manera, mientras que la persistencia del guaraní podría leerse como resistencia a la asimilación lingüística hacia la lengua colonial, la política lingüística paraguaya fomenta la asimilación ideológica. La conciencia lingüística ha sido colonizada con la ideología occidental hegemónica del "estándar monoglósico" purificado, perpetuada por una educación, que aunque sea bilingüe, construye las lenguas educacionales a partir de su diferencia con la manera de hablar de los "otros" del sujeto moderno.

\section{REFERENCIAS BIBLIOGRÁFICAS}

Auer, Peter (coord.). 1998. Code-switching in conversation: Language, interaction, and identity. London \& New York: Routledge. 
Bauman, Richard y Charles L. Briggs. 1990. Poetics and Performances as Critical Perspectives on Language and Social Life. Annual Review of Anthropology 19 (1): 59-88. 2003. Voices of Modernity. Language Ideologies and the Politics of Inequality. Cambridge: Cambridge University Press.

BoIdin, CAPUCINE. 2000. ¿Jopara? ¿jehe'a? Acción. Revista Paraguaya de reflexión y diálogo 32 (206): 28-31.

Bourdieu, Pierre. 1985 [1982]. ¿Qué significa hablar? La economía de los intercambios lingüísticos. Madrid: Akal.

CAdogan, León. 1958. En torno al bilingüismo en el Paraguay. Revista de Antropologia (São Paulo) 6 (1): 23-30.

1990. The Scholastic Point of View. Cultural Anthropology 5 (4): 380-391.

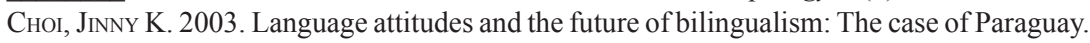
International Journal of Bilingual Education and Bilingualism 6 (2): 81-94.

Comaroff, Jean y John L. Comaroff. 1991. Of Revelation and Revolution, Volume 1. Christianity, Colonialism, and Consciousness in South Africa. Chicago: University of Chicago Press.

Comisión Nacional de BilingüIsmo (coord.). 1997. Ñane ñe'ê Paraguái/Paraguay Bilingüe. Políticas lingüisticas y educación bilingüe. Asunción: Fundación en Alianza.

Corvalán, Graziella. 1981. El bilinguismo en la educación en el Paraguay: ¿es creativo u opresivo? Revista Paraguaya de Sociología 18 (52): 179-200.

1999. Políticas lingüísticas, integración y educación en el Paraguay. Nemitỹ. Revista Bilingüe de Cultura 37: 18-24.

Deleuze, Gilles y Félix Guattari. 1994 [1980]. Mil Mesetas. Capitalismo y esquizofrenia. Valencia: Pre-Textos.

Derrida, JACQues. 1971 [1967]. De la gramatología. Buenos Aires: Siglo XXI Editores.

Ferguson, Charles. 1959. Diglossia. Word 15: 325-340.

Fishman, Joshua A. 1967. Bilingualism with and without diglossia; diglossia with and without bilingualism. Journal of Social Issues 23 (2): 29-38.

Galeano Olivera, David A. 2004. "El jehe'a en la Educación: Sinónimo de Mediocridad". Presentado en el I Congreso departamental de la lengua guaraní en la educación bilingüe, Ateneo de Lengua y Cultura Guaraní, Villarrica, Paraguay, 13 de noviembre de 2004.

Geertz, Clifford. 1973 [1966]. Religion as a Cultural System. En The Interpretation of Cultures, pp. 87-125. New York: Basic Books.

Giddens, ANTHONy. 1979. Central problems in social theory: Action, structure and contradiction in social analysis. Berkeley \& Los Angeles: University of California Press.

DE Granda, Germán. 1980/81. Actitudes sociolingüísticas en el Paraguay. Boletín de Filología 31: 787-805.

1988. Sociedad, Historia y Lengua en el Paraguay. Bogotá: Caro y Cuervo.

1996. Español paraguayo y guaraní criollo. Un espacio para la convergencia lingüística. Signo \& Seña 6: 181-194.

DE GuARANIA, Félix. 2008. El idioma guaraní como resistencia e identidad cultural, entrevistado por Fátima Pérez y Lea Schvartzman. Boletín oficial del Centro Cultural de la República El Cabildo 1 (2): 18-19.

Gynan, Shaw N. 1997. Sociolingüística actual del bilingüismo paraguayo. En Comisión Nacional de Bilingüismo (coord.). Nane ñe'ẽ Paraguái/Paraguay Bilingüe. Políticas lingüisticas y educación bilingüe, pp. 24-31. Asunción: Fundación en Alianza.

1998. Attitudinal dimensions of Guarani-Spanish bilingualism in Paraguay. Southwest Journal of Linguistics 17 (2): 35-57. 
2001. Language Planning and Policy in Paraguay. Current Issues in Language Planning 2 (1): 53-118.

2004. Paraguayan Attitudes toward Standard Guaraní and Spanish. Trans: InternetZeitschrift für Kulturwissenschaften 15: http://www.inst.at/trans/15Nr/06_1/gynan15. htm (27.10.2009).

2007. The Language Situation in Paraguay: An Update. En Richard B. Baldauf, Jr. \& Robert B. Kaplan (eds.). Language Planning and Policy in Latin America: Ecuador, Mexico and Paraguay, pp. 284-301. Clevedon: Multilingual Matters.

HaUCK, JAN DAVID. 2009. Language Under Construction. Bilingualism in Paraguay and some unsettled thoughts about language. Berlin: Weißensee Verlag.

Hill, Jane H. y Kenneth C. Hill. 1986. Speaking Mexicano: Dynamics of Syncretic Language in Central Mexico. Tucson, AZ: University of Arizona Press.

Irvine, Judith T. 1989. When Talk Isn't Cheap: Language and Political Economy. American Ethnologist 16 (2): 248-267.

Kallfell, Guido. 2010. Grammatik des Jopara: Gesprochenes Guarani und Spanisch in Paraguay. Tesis de doctorado. Münster: Westfälische Wilhelms-Universität.

KrämER, SyBILLE. 2001. Sprache, Sprechakt, Kommunikation. Sprachtheoretische Positionen des 20. Jahrhunderts. Frankfurt: Suhrkamp.

Kroskrity, Paul V. 1998. Arizona Tewa Kiva Speech as a Manifestation of a Dominant Language Ideology. En Bambi B. Schieffelin, Kathryn A. Woolard, \& Paul V. Kroskrity (eds.). Language Ideologies. Practice and Theory, pp. 103-122. New York \& Oxford: Oxford University Press.

1999. Identity. Journal of Linguistic Anthropology 9 (1-2): 111-114.

2009. Embodying the Reversal of Language Shift. Agency, Incorporation, and Language Ideological Change in the Western Mono Community of Central California. En Paul V. Kroskrity y Margaret C. Field (eds.). Native American Language Ideologies. Beliefs, Practices, and Struggles in Indian Country, pp. 190-210. Tucson: University of Arizona Press.

2010. Language ideologies — Evolving perspectives. En Jürgen Jaspers, Jan-Ola Östman y Jef Verschueren (eds.). Society and Language Use. Handbook of Pragmatics Highlights, pp. 192-211. Amsterdam: John Benjamins Publishing Company.

Kroskrity, Paul V. y Margaret C. Field (eds.). 2009. Native American Language Ideologies. Beliefs, Practices, and Struggles in Indian Country. Tucson: University of Arizona Press.

Labov, William. 1966. The Social Stratification of English in New York City. Washington, D.C.: Center for Applied Linguistics.

Latour, BRuno. 2007 [1991]. Nunca fuimos modernos. Ensayo de antropología simétrica. Buenos Aires: Siglo XXI Editores.

LipPI-Green, Rosina. 1997. English With an Accent: Language, Ideology, and Discrimination in the United States. London: Routledge.

Lustig, Wolf. 1999. Chácore purahéi — canciones de guerra. Literatura popular en guaraní e identidad nacional en el Paraguay. En Barbara Potthast, Karl Kohut y Gerd Kohlhepp (eds.). El espacio interior de América del Sur. Geografia, historia, politica, cultura, pp. 363-380. Frankfurt \& Madrid: Vervuert/Americana Eystettensia.

Makihara, Miki. 2007. Linguistic Purism in Rapa Nui Political Discourse. En Miki Makihara y Bambi B. Schieffelin (eds.). Consequences of Contact. Language Ideologies and Sociocultural Transformations in Pacific Societies, pp. 49-69. Oxford: Oxford University Press.

Makihara, Miki y Bambi B. SchiefFelin (eds.). 2007. Consequences of contact: Language ideologies and sociocultural transformations in Pacific societies. Oxford: Oxford University Press. 
Melià, Bartomeu. 1973. Diglosia en el Paraguay o la comunicación desequilibrada. Suplemento Antropológico 8: 133-140. CEADUC.

1986. El Guaraní conquistado y reducido. Ensayos de etnohistoria. Asunción:

1992. La lengua guaraní del Paraguay. Madrid: Editorial Mapfre.

Mignolo, Walter. 2000. Local Histories/Global Designs: Coloniality, Subaltern Knowledges, and Border Thinking. Princeton: Princeton University Press.

Milroy, James y Lesley Milroy. 1985. Authority in Language: Investigating Language Prescription and Standardisation. London: Routledge and Kegan Paul.

Ministerio de Educación y Cultura del Paraguay (MEC). 2001. El guaraní mirado por sus hablantes. Investigación relativa a las percepciones sobre el guaraní. Asunción: Ministerio de Educación y Cultura.

2006. La Educación Bilingüe en la Reforma Educativa Paraguaya. Asunción: Ministerio de Educación y Cultura.

Mortimer, Katherine. 2006. Guaraní Académico or Jopará? Educator Perspectives and Ideological Debate in Paraguayan Bilingual Education. Working Papers in Educational Linguistics 21 (2): 45-71.

Ochs, Elinor. 1990. Indexicality and socialization. En James W. Stigler, Richard A. Shweder y Gilbert H. Herdt (eds.). Cultural Psychology. Essays on comparative human development, pp. 287-308. Cambridge: Cambridge University Press.

Peirce, Charles S. 1998 [1893-1913]. The Essential Peirce: Selected Philosophical Writings, Volume 2. Bloomington IN: Indiana University Press.

Penner, Hedy, Malvina Segovia, Rosana Spinzi y Limpia Esteche. 2009. Los rasgos del castellano paraguayo y sus paradigmas epistemológicos. Suplemento Antropológico 44 (2): 387-464.

Potthast, Barbara. 1999. El mestizaje del Paraguay como identidad nacional y mito nacionalista. En Barbara Potthast, Karl Kohut y Gerd Kohlhepp (eds.). El espacio interior de América del Sur. Geografia, historia, politica, cultura, pp. 345-362. Frankfurt \& Madrid: Vervuert/Americana Eystettensia.

Paraguay. 1992. Constitución de la República de Paraguay. Asunción.

de Saussure, Ferdinand. 1945 [1916]. Curso de lingüistica general. Buenos Aires: Losada.

Silverstein, Michael. 1976. Shifters, Linguistic Categories, and Cultural Description. En Keith H. Basso y Henry A. Selby (eds.) Meaning in Anthropology, pp. 11-55. Albuquerque: University of New Mexico Press.

1979. Language structure and linguistic ideology. En Paul R. Clyne, William F. Hanks y Carol L. Hofbauer (eds.). The Elements: A Parasession on Linguistic Units and Levels, pp. 193-247. Chicago: Chicago Linguistic Society.

1996. Monoglot 'Standard' in America: Standardization and Metaphors of Linguistic Hegemony. En Donald Brenneis y Ronald Macaulay (eds.). The Matrix of Language. Contemporary Linguistic Anthropology, pp. 284-306. Boulder: Westview Press.

SolÉ, Yolanda Russinovich. 1996. Language, affect, and nationalism in Paraguay. En Ana Roca y John B. Jensen (eds.). Spanish in contact: Issues in bilingualism, pp. 93-111. Somerville, MA: Cascadilla Press.

Spivak, Gayatri ChaKravorty. 1988. Can The Subaltern Speak? En Cary Nelson y Lawrence Grossberg (eds.). Marxism and the Interpretation of Culture, pp. 271-313. Urbana \& Chicago: University of Illinois Press.

Steckbauer, Sonja M. 1999. La situación del guaraní en el Paraguay actual. En Barbara Potthast, Karl Kohut y Gerd Kohlhepp (eds.). El espacio interior de América del Sur. Geografia, historia, politica, cultura, pp. 381-400. Frankfurt \& Madrid: Vervuert/ Americana Eystettensia. 
Whiteley, Peter. 2003. Do 'Language Rights' Serve Indigenous Interests? Some Hopi and Other Queries. American Anthropologist 105 (4): 712-722.

Williams, Raymond. 1977. Marxism and Literature. Oxford: Oxford University Press.

WoOlard, Kathryn A. 1998. Introduction. Language Ideology as a Field of Inquiry. En Bambi B. Schieffelin, Kathryn A. Woolard y Paul V. Kroskrity (eds.). Language Ideologies. Practice and Theory, pp. 3-47. New York \& Oxford: Oxford University Press.

2004. Codeswitching. En Alessandro Duranti (ed.). A Companion to Linguistic Anthropology, pp. 73-94. Malden, MA: Blackwell.

Woolard, Kathryn A. y Bambi B. Schieffelin. 1994. Language Ideology. Annual Review of Anthropology 23 (1): 55-82. 